\title{
Conocimientos de los padres sobre la salud bucal de niños preescolares: desarrollo y validación de un instrumento
}

Parental knowledges of preschool children's oral health: development and validation of the instrument Ana Cecilia Cupé-Araujo ${ }^{1}$, Carmen Rosa García-Rupaya ${ }^{2}$

\section{RESUMEN}

Objetivo: Validar un instrumento para medir los conocimientos sobre salud bucal en madres y padres de niños que asisten a Instituciones Educativas Iniciales en zonas urbano marginales de la provincia de Lima en el año 2012. Material y métodos: Estudio observacional, prospectivo, descriptivo y transversal. La principal técnica de recolección de datos fue la encuesta, mediante un cuestionario estructurado. Resultados: La obtención de la validez de contenido se obtuvo mediante la prueba estadística V de Aiken, la reproducibilidad, fue calculada con el CCI en una muestra de 30 madres y/o padres de familia, para la validez de constructo se analizaron las respuestas del cuestionario de 312 padres y/o madres de familia con el análisis factorial. Finalmente, se evaluó la confiabilidad de los resultados obtenidos con la prueba estadística Alfa de Cronbach. Dentro de los resultados se pudo observar que se obtuvo una validez de contenido de 0.95 , reproducibilidad de 0.89 , validez de constructo el resultado menor de 0.36 en el ítem de amamantamiento nocturno y el más elevado 0.77 en el ítem de lactancia materna y finalmente la confiabilidad de 0.64. Conclusión: El instrumento es seguro y confiable en la medición de los conocimientos sobre salud bucal en los padres de familia de niños que asisten al nivel inicial.

PALABRAS CLAVE: Conocimiento, validación, preescolar, salud bucal. (DeCS, BIREME)

Facultad de Ciencias de la Salud, Universidad Norbert Wiener. Lima, Perú.

Sociedad Peruana de Endodoncia. Lima, Perú.

Especialista en Odontopediatría.

Especialista en Endodoncia.

Magister en Estomatología con mención en Odontología Pediátrica.

Magister en Estomatología. 


\section{SUMMARY}

Objetives: Validate an instrument to measure the knowledge on mouth health in parents of children who go at Institutions Educational Initial in zones urbano marginales of the province of Lima in the year 2012. Methods: Type of study was observacional, descriptive and transverse, the compilation of information was the survey, by means of a structured questionnaire. Results: Proceeded to the obtaining of the validity of content by means of the statistical test $V$ de Aiken, there was evaluated the reproducibility, which was calculated by the CCI in a sample of 30 parents, for the validity of constructo there were analyzed the answers of the questionnaire of 312 parents and / or family mothers by the analysis factorial. Finally, there was evaluated the reliability of the results obtained with the statistical Test of Cronbach's Alpha. Inside the results we could observe that validity obtained a validity of content of 0.95 , reproducibilidad of 0.89 , of constructo the minor result of 0.36 in the article and highest of night nursing 0.77 in the article of mother lactation and finally the Reliability of 0.64. Conclusion: The instrument is safe and reliable in measuring oral health knowledge in parents of children attending the initial level.

KEYWORDS: Knowledge, validation, pre-school, oral health. (MeSH, NLM)

\section{INTRODUCCIÓN}

Actualmente, para la decisión de tratamiento y manejo de las enfermedades bucales, no sólo es importante conocer los indicadores clínicos bucales de los individuos antes de realizar cualquier tipo de intervención, sino también saber los niveles de conocimientos que los pacientes o sus responsables tienen sobre la etiología y prevención, así como las prácticas diarias de higiene y la actitud frente a la misma (1).

Los padres desempeñan un papel crucial en el soporte emocional, necesidad de adaptación comportamental frente a enfermedades o tratamientos y en el financiamiento de los costos en la salud de los niños (2). Para que haya entonces salud bucal, los padres deben ser educados y concientizados para sensibilizar tempranamente a sus hijos en la adquisición de comportamientos que permitan un buen desenvolvimiento de hábitos de salud bucal $(3,4)$.

Las acciones para proteger la salud bucal de los niños se basan en una constante participación de los padres o tutores, en programas del área maternoinfantil y al trabajo de pediatría de una manera general (5). Sin embargo, existe una gran dificultad para conseguir la colaboración de los padres, para la atención o realización de maniobras preventivas en el ámbito doméstico (6).
La adquisición de información y desarrollo de habilidades es parte de la educación en salud, para estimular el cambio de comportamiento y actitud del individuo, beneficiando la salud de manera general (7). Actualmente la literatura sugiere que la educación en salud bucal sea lo más precoz posible, ya que durante el periodo gestacional la mujer se encuentra más susceptible a adquirir conocimientos sobre cuidados para con su bebé, y consecuentemente también receptiva a entender el valor de la buena salud bucal, así la prevención iniciada precozmente se torna más efectiva y fácil de realizar. Por ese motivo es importante que el núcleo familiar sea un punto clave en la prevención de enfermedades, como conductor de hábitos alimenticios de la familia y formadora de conceptos sobre salud. Desde el inicio del siglo, algunos profesionales del área odontológica han demostrado preocupación en desmitificar la creencia popular (8-10).

La caries dental precoz es un grave problema de salud pública, ya que los niños que presentan caries en la primera infancia tienen mayor probabilidad de desarrollar caries tanto en dentición decidua como en permanente, como también dolor al momento de comer (11). Otras consecuencias de caries dental en la vida de los niños incluyen hospitalizaciones y visitas a la atención de emergencia (12), aumento en los costos del tratamiento (13), riesgo de atraso del crecimiento físico y aumento de peso (14), e impacto negativo en la calidad de vida relacionada a la salud 
bucal (15).

Los datos de salud bucal en el mundo indican que la caries dental presenta una prevalencia disminuida en la mayoría de los países; por lo tanto, el fenómeno de polarización de esa enfermedad va demostrando que tal descenso no ocurre de forma homogénea, ya que aún existe un gran número de personas pertenecientes a grupos minoritarios, socialmente desprotegidos, con mayor vulnerabilidad a las enfermedades bucales, por ser más susceptibles o estar más expuestos a factores de riesgo $(16,17)$. La literatura destaca la importancia de la educación para la salud bucal de preescolares, como refuerzos para la adquisición de actitudes y hábitos saludables de toda la familia, ya que por medio de las escuelas, los padres/responsables toman conocimiento sobre medidas saludables. Los padres y/o responsables de los niños, muchas veces no poseen toda la información necesaria sobre los cuidados para mantener la salud bucal de los niños en edad preescolar. Capacitándolos será posible mayor eficiencia y técnica en el trabajo de promoción de la salud bucal (18). Por tal motivo, este estudio tuvo como objetivo desarrollar y validar un instrumento para medir los conocimientos sobre salud bucal en madres y padres de niños preescolares.

\section{MATERIAL Y MÉTODOS}

El diseño de la investigación fue de tipo transversal. La población fue el total de padres de familia con niños en edad preescolar (3 a 5 años) matriculados y con asistencia regular de cinco Instituciones Educativas Públicas. Para la obtención del tamaño de la muestra en la etapa de constructo y confiabilidad se utilizó la fórmula de estimar una proporción que nos dio como resultado 190 padres y/o madres de familia a encuestar (19). El tamaño de la muestra se incrementó a 312 por las facilidades que nos fue brindada por las Instituciones Educativas Iniciales. Se seleccionó al azar 4 distritos de Lima Metropolitana y los distritos seleccionados fueron: Cercado de Lima, con el Instituto de Educación Inicial (I. E. I.) Nuestra Señora de Monserrat con 150 niños; el distrito de Puente Piedra, con el I. E. I. Micaela Bastidas con 50 niños y en el distrito de Villa María del Triunfo con el I. E. I. Universo con 30 niños, Santa Rosa de Lima con 32 niños y el I. E. I. Niño Jesús con 50 niños. Los criterios de inclusión fueron: padres que tuvieron hijos en edad preescolar, que firmaron el consentimiento informado, que presentaron buen estado físico y mental, y que deseaban participar en el estudio.

La investigación tuvo dos momentos importantes:

\section{Coordinación con las Instituciones Educativas Iniciales}

Se procedió a establecer contacto con los directores de las I. E. I., con la entrega de los documentos pertinentes, con el fin de obtener los permisos correspondientes. Luego los padres firmaron el consentimiento informado para ser parte de la investigación.

\section{Validación del cuestionario}

Validación de Contenido: la principal técnica de recolección de datos fue la encuesta, mediante un cuestionario estructurado elaborado por el investigador, éste contenía 20 preguntas sobre: prevención, caries dental, dieta e higiene; con cuatro alternativas de respuesta, el puntaje fue de 0 a 20 según el Ministerio de Educación del Perú; a su vez fue revisado por 4 odontólogos especialistas en odontopediatría, y 3 odontólogos especialistas en metodología de la investigación, todos con más de 10 años de experiencia en el dichas áreas y validación. La prueba que se utilizó fue el Coeficiente de Validez (V de Aiken).

Reproducibilidad: Se evaluó una muestra de 30 padres y/o madres de niños que asistían de forma regular al I. E. I. Rayito de Luz, institución diferente a la muestra seleccionada para el constructo. El tamaño para la reproducibilidad tomo en cuenta lo estimado por Arribas (20). Luego de aplicada la primera encuesta ( $\mathrm{n}=20$ preguntas), se volvió a aplicar la misma a los padres de familia a los 15 días, pero cambiando el orden de las preguntas para evitar el sesgo de memoria (fiabilidad test-retest). La fiabilidad, fue calculada con el Coeficiente de Correlación Intraclase (CCI).

Validez de constructo: Se evaluaron los conocimientos sobre salud bucal a 312 madres y/o padres de familia de los I. E. I. Micaela Bastidas, Nuestra Señora de Monserrat, Universo, Santa Rosa de Lima y Niño Jesús con el cuestionario elaborado 
y corregido por el juicio de expertos. Finalmente, se analizaron las respuestas y se aplicó la prueba de análisis factorial.

Confiabilidad: Los resultados obtenidos de la muestra sirvieron para determinar la confiabilidad del instrumento mediante la prueba estadística de Alfa de Cronbach (20).

\section{RESULTADOS}

Se diseñó un cuestionario para luego proceder con la validación del mismo, para esto se realizó la validación del contenido con el juicio de 7 expertos en el área, se utilizó el método estadístico de la V de Aiken, para la obtención de los resultados, obteniendo como resultado final, un valor de 0.95. La reproducibilidad (Test.retest) se realizó en una muestra conformada por 30 madres $\mathrm{y} / \mathrm{o}$ padres de familia del I. E. I. Rayito de Luz y se utilizó el Análisis Coeficiente de Correlación Interclase con un resultado de 0.89. Posteriormente el estudio se realizó en 312 padres de familia: 260 del sexo femenino y 52 del sexo masculino.

En la validez de constructo, se utilizó el análisis factorial con la obtención más baja (0.361) en la pregunta sobre amamantamiento nocturno, y obteniendo la puntuación más alta $(0.775)$ en la pregunta sobre lactancia materna. Mientras que para la evaluación de la confiabilidad, se aplicó la prueba estadística del Alfa de Cronbach con un resultado que va de regular a bueno de 0.645 .

Cuando observamos la relación entre el nivel de conocimiento sobre salud bucal y el sexo de los padres de familia, se observó que 133 (42,6\%) madres de familia obtuvieron una calificación "buena" (15 a 17) y $14(4,5 \%)$ madres obtuvieron una calificación "deficiente" (0 a 10) en el cuestionario. En el caso de los padres $24(7,7 \%)$ obtuvieron una calificación "buena" (15 a 17) y 3 (1\%) obtuvieron una calificación "deficiente" ( 0 a 10) en el cuestionario sobre salud bucal. No existió asociación estadísticamente significativa entre el nivel de conocimiento sobre salud bucal y el sexo de los padres (Tabla 1).

Respecto a la relación entre nivel de conocimiento sobre salud bucal y grado de instrucción de las madres de familia: 89 (34,2\%) madres con educación técnica y/o superior obtuvieron una calificación "buena"(15 a 17), y $5(1,9 \%)$ obtuvieron una calificación "deficiente" (0 a 10); en el caso de las madres con educación secundaria $43(16,5 \%)$ obtuvieron una calificación "buena"(15 a 17) y $2(0,8 \%)$ una calificación "deficiente" (0 a 10). Finalmente, en el caso de las madres con educación primaria $5(1,9 \%)$ obtuvieron una calificación "deficiente", al igual que $2(0,8 \%)$ con ninguna educación. Se observó una asociación estadísticamente significativa entre el nivel de conocimiento sobre salud bucal y grado de instrucción de las madres de familia encuestadas (Tabla 2).

Respecto a la relación entre el nivel de conocimiento sobre salud bucal y grado de instrucción de los padres de familia: $21(40,4 \%)$ padres con educación técnica y/o superior obtuvieron una calificación "buena"(15 a 17), y $1(1,9 \%)$ obtuvo una calificación "deficiente" (0 a 10); los padres con educación secundaria 3 $(5,8 \%)$ obtuvieron una calificación "buena"(15 a 17) y "regular" (11 a 14); no existió ningún padre de

Tabla 1. Nivel conocimiento sobre salud bucal en madres y padres de niños que asisten a Instituciones Educativas Iniciales en zonas urbano marginales de la provincia de Lima, año 2012.

\begin{tabular}{ccccc}
\hline & \multicolumn{3}{c}{ Sexo } & \multicolumn{2}{c}{ Padres } \\
Conocimiento sobre Salud Bucal & \multicolumn{2}{c}{ Madres } & n & \% \\
\hline Deficiente & n & \% & 3 & 1 \\
Regular & 14 & 4.5 & 18 & 5.8 \\
Bueno & 62 & 19.9 & 24 & 7.7 \\
Muy Bueno & 133 & 42.6 & 7 & 2.2 \\
Total & 51 & 16.3 & 52 & 16.7 \\
\hline
\end{tabular}

Chi cuadrado

$\mathrm{p}=0.381$ 
Tabla 2. Nivel de conocimiento sobre salud bucal en madres de niños que asisten a Instituciones Educativas Iniciales en zonas urbano marginales de la provincia de Lima con relación al grado de instrucción, año 2012.

\begin{tabular}{cccccccccc}
\hline $\begin{array}{c}\text { Conocimiento sobre Salud } \\
\text { Bucal }\end{array}$ & \multicolumn{1}{c}{$\begin{array}{c}\text { Ninguna } \\
\end{array}$} & $\begin{array}{c}\text { Educación } \\
\text { Primaria }\end{array}$ & \multicolumn{2}{c}{$\begin{array}{c}\text { Educación } \\
\text { Secundaria }\end{array}$} & $\begin{array}{c}\text { Educación Técnica } \\
\text { y/o Superior }\end{array}$ \\
& $\mathbf{n}$ & $\mathbf{n}$ & $\mathbf{\%}$ & $\mathbf{n}$ & $\mathbf{\%}$ & $\mathbf{n}$ & $\mathbf{\%}$ \\
\hline Deficiente & 2 & 0.8 & 5 & 1.9 & 2 & 0.8 & 5 & 1.9 \\
Regular & 0 & 0 & 4 & 1.5 & 23 & 8.8 & 35 & 13.5 \\
Bueno & 0 & 0 & 1 & 0.4 & 43 & 16.5 & 89 & 34.2 \\
Muy Bueno & 0 & 0 & 2 & 0.8 & 10 & 3.8 & 39 & 15 \\
\hline
\end{tabular}

Chi cuadrado

$\mathrm{p}=0.000$

Tabla 3. Nivel de conocimiento sobre salud bucal en padres de niños que asisten a Instituciones Educativas Iniciales en zonas urbano marginales de la provincia de Lima con relación al grado de instrucción, año 2012.

\begin{tabular}{|c|c|c|c|c|c|c|c|c|}
\hline \multirow{3}{*}{ Conocimiento sobre Salud Bucal } & \multicolumn{8}{|c|}{ Padres } \\
\hline & \multicolumn{2}{|c|}{ Ninguna } & \multicolumn{2}{|c|}{$\begin{array}{c}\text { Educación } \\
\text { Primaria }\end{array}$} & \multicolumn{2}{|c|}{$\begin{array}{l}\text { Educación } \\
\text { Secundaria }\end{array}$} & \multicolumn{2}{|c|}{$\begin{array}{c}\text { Educación } \\
\text { Técnica y/o } \\
\text { Superior }\end{array}$} \\
\hline & $\mathbf{n}$ & $\%$ & $\mathbf{n}$ & $\%$ & $\mathbf{n}$ & $\%$ & n & $\%$ \\
\hline Deficiente & 1 & 1.9 & 0 & 0 & 1 & 1.9 & 1 & 1.9 \\
\hline Regular & 0 & 0 & 0 & 0 & 3 & 5.8 & 15 & 28.8 \\
\hline Bueno & 0 & 0 & 0 & 0 & 3 & 5.8 & 21 & 40.4 \\
\hline Muy Bueno & 0 & 0 & 0 & 0 & 2 & 3.8 & 5 & 9.6 \\
\hline
\end{tabular}

familia con educación primaria dentro de la muestra $(0 \%)$. Finalmente, solo $1(1,9 \%)$ que no presentaba educación, obtuvo una calificación "deficiente". Se observó una asociación estadísticamente significativa entre el nivel de conocimiento sobre salud bucal y grado de instrucción de los padres de familia (Tabla $3)$.

Al evaluar la edad de las madres y el nivel de conocimiento sobre salud bucal, se observó que 106 $(40,8 \%)$ de las madres adultas jóvenes obtuvieron una calificación "buena"(15 a 17) y $9(3,5 \%)$ obtuvieron una calificación "deficiente" ( 0 a 10$)$; en el caso de las madres adultas maduras $27(10,4 \%)$ obtuvieron una calificación "buena" (15 a 17) y $5(1,9 \%)$ una calificación "deficiente" (0 a 10), no existió diferencia estadísticamente significativa entre edad de las madres de familia y nivel de conocimiento sobre salud bucal (Tabla 4).

Cuando se evaluó la edad de los padres de familia y el nivel de conocimiento sobre salud bucal, se observó que 20 (38,5\%) de los padres adultos jóvenes obtuvieron una calificación "buena" (15 a 17) y 2 $(3,8 \%)$ obtuvieron una calificación "deficiente" $(0$ a 10); en el caso de los padres adultos maduros 10 $(19,2 \%)$ obtuvieron una calificación "regular" (11 a 14) y $1(1,9 \%)$ una calificación "deficiente" (0 a 10) y otro una calificación "muy buena". Encontrándose que existió diferencia estadísticamente significativa entre la edad de los padres de familia y el nivel de conocimiento sobre salud bucal. 
Tabla 4. Nivel de conocimiento sobre salud bucal en madres de niños que asisten a Instituciones Educativas Iniciales en zonas urbano marginales de la provincia de Lima con relación a su edad, año 2012.

\begin{tabular}{lccccc}
\hline & \multicolumn{4}{c}{ Madres } \\
Conocimiento sobre Salud Bucal & \multicolumn{2}{c}{$\begin{array}{c}\text { Adulto } \\
\text { Joven }\end{array}$} & \multicolumn{2}{c}{ Adulto } \\
& & Maduro \\
& n & \% & n & \% \\
\hline & Deficiente & 9 & 3.5 & 5 & 1.9 \\
& Regular & 49 & 18.8 & 13 & 5 \\
& Bueno & 106 & 40.8 & 27 & 10.4 \\
& Muy Bueno & 41 & 15.8 & 10 & 3.8 \\
\hline Chi cuadrado & & & & & \\
$\mathrm{p}=0.59$ & & & & &
\end{tabular}

Tabla 5. Nivel de conocimiento sobre salud bucal en padres de niños que asisten a Instituciones Educativas Iniciales en zonas urbano marginales de la provincia de Lima con relación a su edad, año 2012.

\begin{tabular}{ccccc}
\hline & \multicolumn{5}{c}{ Padres } \\
Conocimiento sobre Salud Bucal & Adulto Joven & Adulto Maduro \\
& n & \% & n & \% \\
\hline Deficiente & 2 & 3.8 & 1 & 1.9 \\
Regular & 8 & 15.4 & 10 & 19.2 \\
Bueno & 20 & 38.5 & 4 & 7.7 \\
Muy Bueno & 6 & 11.5 & 1 & 1.9 \\
\hline
\end{tabular}

Chi cuadrado

$\mathrm{p}=0.04$

\section{DISCUSIÓN}

La importancia del presente trabajo se centra en poder brindar a la comunidad científica un instrumento validado en Lima, que permita estudiar con mayor certeza los conocimientos sobre salud bucal en padres con niños preescolares. Como aseguran Gómez y col. (21) es importante estudiar estos conocimientos en los padres de niños pequeños, a fin de enfatizar la promoción de la salud bucal en la primera infancia; ello debido a que un nivel de conocimiento bajo se asocia con mayores índices de enfermedades bucales, tales como: caries dental; ya que existe relación significativa entre prevalencia de caries dental en preescolares y el nivel de conocimiento de sus madres sobre salud bucal, según lo reportado por Pisconte y Portocarrero (22).
Por lo tanto, al conocer los niveles de conocimientos de las madres/padres sobre salud bucal; implica asegurarse que un niño preescolar tenga un padre con buen conocimiento sobre medidas preventivas para evitar enfermedades bucales, tal como refiere Schroth (23) quien observó que padres con mayor conocimiento sobre salud bucal tuvieron niños con menos caries dental.

El principal aporte de esta investigación es que permite ayudar a los padres y a sus hijos, a partir de la medición de su nivel de conocimiento sobre salud bucal. Teniendo en cuenta que los hábitos de higiene bucal se establecen durante los primeros años de vida, siendo los padres y principalmente la madre quien funciona como modelo de aprendizaje (24).

En la presente investigación, primero se determinó la validez del contenido, a través de la prueba de 
V de Aiken, encontrándose en la mayoría de las preguntas un resultado alto, interpretándose como claridad, congruencia y comprensión de los ítems que constituyen el cuestionario. En segundo lugar, se verificó la reproducibilidad a través del Test-retest el cual se realizó en una muestra conformada por 30 padres de familia de un colegio diferente al cual se le aplicó el cuestionario en la fase empírica; para esto se utilizó el Coeficiente de Correlación Intraclase, obteniendo un resultado de 0,89 que corresponde a una fuerza de concordancia muy buena.

Para la validez del constructo se utilizó el análisis factorial, obteniéndose valores que oscilan entre 0,36 hasta 0,77 ; la puntuación más baja se obtuvo en la pregunta sobre amamantamiento nocturno y la más alta en la pregunta sobre lactancia materna. Para analizar la confiabilidad se realizó la prueba estadística Alfa de Cronbach, obteniéndose como resultado 0,645 que va de "regular" a "bueno".

Cuando se relaciona el nivel de conocimiento con el grado de instrucción, se obtiene que las madres con educación superior obtuvieron un nivel de conocimiento sobre salud bucal bueno $(34,2 \%)$ al igual que los padres $(40,4 \%)$. Esto a su vez se puede ver influenciado por el estatus socioeconómico, tal como lo obtenido en el estudio de Theodoro y col. (25) quienes concluyen que mientras la madre presentaba un mejor estatus socioeconómico, poseen un mejor grado de conocimiento sobre salud bucal.

Al evaluar el nivel de conocimiento de las madres en relación a la edad, no se encontró asociación entre las variables; sin embargo, el mayor porcentaje con un nivel de conocimiento bueno $(40,8 \%)$ estuvo en el grupo de adulto joven; esto podría deberse más al número de individuos por grupo que a la influencia de la edad sobre el nivel de conocimiento. Contrario a ello, en el grupo de padres si se obtuvo asociación estadísticamente significativa entre las variables $(\mathrm{p}<$ $0,05)$ encontrándose que el nivel de conocimientos bueno estuvo en el grupo de adulto joven con $38,5 \%$, pudiendo deberse este valor a la diferencia entre el número de individuos por grupo.

Dentro de las limitaciones evidenciadas en la fase empírica del estudio se observó la falta de cooperación de los padres y la ausencia de un espacio adecuado para el llenado de los cuestionarios; por lo cual, serán necesarias tomar en cuenta estas recomendaciones para futuras investigaciones. Particularmente, es esencial desarrollar la validez y confiabilidad de un instrumento cuando se trata de un intento para medir el nivel de conocimiento sobre salud bucal de las madres y padres; por lo general esto se utiliza para la comparación de las respuestas de esos individuos. La corroboración adicional resulta al aplicar una escala de calificación según el Ministerio de Educación AD (muy bueno) 18-20 puntos, A (bueno) 15-17 puntos, $\mathrm{B}$ (regular) 11-14 puntos y C (malo) 0-10 puntos.

Los resultados obtenidos y las ventajas señaladas motivan a profundizar en el proceso de validación de este instrumento y abordar la siguiente etapa; es decir un estudio de validación incorporando una muestra aún más representativa de la población peruana, permitiendo de esta manera proponer un modelo de intervención educativa para la promoción y prevención de la salud bucal. Por último, es importante destacar que la adaptación y validación de este instrumento en nuestro país, nos da la posibilidad de contar con una herramienta sencilla y así mejorar o reforzar la atención primaria en la salud bucal.

En conclusión, se comprobó que el instrumento probado es válido y confiable, que hubo asociación estadísticamente significativa entre el nivel de conocimiento sobre salud bucal y grado de instrucción de las madres y padres. Mostrando que a medida que la madre o el padre presentaba un nivel de educación mayor, presentaba un mejor nivel de conocimiento sobre salud bucal.

\section{Correspondencia:}

Ana Cecilia Cupé-Araujo

Mz T Lote 13, Urbanización El Golf de Huampani. Lima, Perú.

Correo electrónico: anace1250@hotmail.com

\section{REFERENCIAS BIBLIOGRÁFICAS}

1. González-Martínez F, Sierra C, Morales L. Conocimientos, actitudes y prácticas en salud bucal de padres y cuidadores en hogares infantiles, Colombia. Salud Pública Mex. 2011; 53(3):247-57.

2. Mayo NE, Wood-Dauphinee S, Cote R, Gayton D, Carlton J, Buttery J. There's no place like home: an evaluation of early supported discharge for stroke. Stroke 2000; 31(5):1016-23. 
3. World Health Organization [Internet]. The World Health Report 2003:Continuous Improvement of Oral healh in the 21st Century-The approach of the WHO Global Oral Health Programme. [Citado el 10 de febrero del 2010] Disponible en: <http://www.who.int/ oral_health/media/en/orh_report03_en.pdf $>$.

4. Dias MS. Estudo dos Factores de Risco em Saúde Oral em Crianças Escolarizadas. [Tesis de licenciatura en medicina dentária] Porto: Universidad Fernando Pessoa; 2009.

5. Pinto, GV. Saúde Bucal Coletiva. $4^{\circ}$ edición, São Paulo: Santos, 2000.

6. Baldani MHP, Lopes CMDL, Kriger L, Matsuo T. A Odontologia para bebês no estado do Paraná, Brasil. Perfil do programa de atenção precoce à saúde bucal. J Bras Odontopediatr Odontol Bebê. 2003; 6(31):210-6.

7. Franzin LCS, Bijella MST. Conhecimento de um grupo de mães sobre a saúde bucal de seus filhos. Rev Iberoam Odontopediatr Odontol Bebê. 2005; 8(45):333-41.

8. González-Martínez F, Sierra-Barrios CC, MoralesSalinas LE. Conocimientos, actitudes y prácticas en salud bucal de padres y cuidadores en hogares infantiles, Colombia. Salud Publica Mex.2011; 53(3):247-57.

9. Inocente-Díaz ME, Pachas-Barrionuevo FM. Educación para la salud en odontología. Rev Estomatol. Herediana. 2012; 22(4):232-9.

10. Günther K, Tovo FM, Feldens CA. Avaliação dos conhecimentos sobre saúde bucal referidos por parturientes do Hospital Luterano - ULBRA. Stomatos. 2005; 11(20):6-12.

11. Perez-Luyo AG. ¿Es la caries dental una enfermedad infecciosa y transmisible? Rev Estomatol Herediana. 2009; 19(2):118-24.

12. Ladrillo TE, Hobdell MH, Caviness C. Increasing prevalence of emergency department visits for pediatric dental care 1997-2001. J Am Dent Assoc. 2006; 137(3):379-85

13. Kanellis MJ, Damiano PC, Monamy ET. Medicaid costs associated with the hospitalization of young children for restorative dental treatment under general anesthesia. J Public Health Dent. 2000; 60(1):28-32.

14. Sheiham A. Dental caries affects body weight, growth and quality of life in pre-school children. Br Dent J. 2006; 201(10):625-6.

15. Abanto J, Carvalho TS, Mendes FM, Wanderley MT, Bönecker M, Raggio DP. Impact of oral diseases and disorders on oral health-related quality of life of preschool children. Community Dent Oral Epidemiol. 2011; 39(2):105-14.

16. Ramos-Gómez F. Cuidado dental pediátrico: prevención y protocolos de manejo basados en evaluación de riesgo de caries. En: Perona Miguel de Priego G, Castillo Cevallos JL.Manejo odontológico materno infantil basado en evidencia científica. Madrid: Editorial Ripano; 2012.p.187-97.

17. Gabínio MF, De Araújo MC, Correira F, Vasconcelosb LC, Vasconcelosb LC. Evaluation of an oral health program for children in early childhood. Rev Odonto Ciênc. 2010; 25(4):350-4.

18. Rodriguez CA. Conocimientos de salud bucal de estudiantes de $7^{\circ}$ y $8^{\circ}$ básico de la ciudad de Santiago. [Tesis para optar el título de Cirujano dentista]. Santiago: Universidad de Chile; 2005.

19. Suresh BS, Ravishankar TL, Chaitra TR, Mohapatra AK, Gupta V. Mother's knowledge about pre-school child's oral health J Indian Soc Pedod Prev Dent. 2010; 4(28):282-7.

20. Arribas M. Diseño y validación de cuestionarios. Matronas Profesión. 2004; 5(17):23-9.

21. Gomez AM, Ferreira CG, Leite A, Vasconcelos PF. Percepção materna sobre a higiene bucal de bebês: um estudo no Hospital Alcides Carneiro, Campina GrandePB. Pesq Bras Odontoped Clin Integr, João Pessoa. 2004; 4(3):185-9.

22. Arribas M. Diseño y validación de cuestionarios. Matronas Profesión. 2004; 5(17):23-9.

23. Theodoro D, Gigliotti M, Oliveira T, Da Silva S, Machado MA. Fator socioeconômico e o grau de conhecimento das mães em relação à saúde bucal de bebês. Odontologia Clín Cient. 2007; 6(2):133-7.

24. Piscote EG. Relación entre la prevalencia de caries dental en preescolares y el nivel de conocimiento de sus madres sobre salud dental. Distrito La Esperanza Trujillo. [Tesis para optar el título de Cirujano Dentista]. Trujillo: Universidad Privada Antenor Orego; 2010.

25. Campos L, Rabaldo E, Bez J, Garcia E, Eger BH. Conhecimento da mães de diferentes clases sociais sobre saúde bucal no municipio de Cocal do Sul (SC). Rev Sul-Bras Odontol. 2010; 7(3):287-95.

Recibido: 05/12/2014

Aceptado: 12/05/2015 


\section{CUESTIONARIO}

Fecha:

Edad de su niño(a):

a) 3 años

b) 4 años

c) 5 años

¿Quién responde al cuestionario?
a) Padre

b) Madre

Edad del informante:

Grado de instrucción del padre:
a) Ninguna
b)Educación Primaria
c)Educación

Secundaria

d) Educación Técnica y/o Superior

El cuestionario está elaborado para que usted de acuerdo a su criterio indique una respuesta mediante un aspa (X).

\section{CARIES DENTAL}

1.- ¿Qué es la caries dental?

a.- Es una enfermedad que aparece en los niños desnutridos.

b.- No es una enfermedad

c.- Es una enfermedad causada por falta de higiene bucal y consumo de azúcares.

d.- Es una enfermedad causada por falta de higiene bucal y consumo de frituras.

2.- Los microorganismos que causan la caries dental puede transmitirse por

a) Compartir utensilios

b) Compartir cepillos dentales

c) Besos en la boca

d) Todas las anteriores

3.- ¿Qué es la placa bacteriana dental?

a.- Es una capa dura que se forma en la superficie de los dientes

b.- Es una placa blanda que se forma en la superficie de los dientes

c.- Es el sarro que se forma en los dientes

d.- Es una masa que solo se encuentra en los dientes de los adultos

4.- ¿Son importantes los dientes de leche?

a) Sí, porque guardan espacio para los dientes permanentes

b) No, porque no cumplen ninguna función

c) No, porque al final se van a caer

d) No, porque no son los dientes permanentes

5.- ¿A qué edad los niños tienen todos los dientes de leche?

a) 2 años

b) 4 años

c) 6 años

d) 8 años

\section{PREVENCIÓN}

6.- La primera visita al odontólogo, se recomienda a partir de:
a) A partir de los 2 años
b) Cuando aparece el primer diente de leche
c) Cuando tenemos dientes de adulto
d) Solo cuando existe dolor

7.- ¿Qué beneficios conoce del flúor?

a) Fortalece los dientes y previene la caries

b) Cura los dientes para prevenir las extracciones

c) El flúor tiene una acción blanqueadora en los niños

d) Fortalece al niño para que crezca sano y fuerte

8.- Es necesario visitar al dentista cuando se tiene dientes sanos:

a) $\mathrm{Si}$, para un examen clínico de rutina

b) Solo si tiene dientes chuecos

c) No, ya que como no tiene nada no es necesario

d) Solo voy si mi hijo tiene molestias en la boca

9.- Si su niño pierde un diente de leche, antes del tiempo porque esta con caries ¿cree Ud. que pueda afectar la posición de los dientes?
a) $\mathrm{Si}$
b) Depende, solo si pierde las muelas de leche
c) No
d) Los dientes de leche no son importantes

10.- ¿Qué medidas preventivas conoce usted para combatir la caries dental?
a) El flúor
b) Una correcta higiene bucal
c) Evitar consumo excesivo de azúcares
d) Todas las anteriores

\section{DIETA}

11.- ¿Qué alimentos cree usted que son mejores para tener dientes más sanos?
a) Frutas y vegetales
b) Gaseosas y frutas
c) Frugos y galletas
d) Todas las anteriores

12.- ¿Qué se recomienda que lleve su hijo en la lonchera?

a) Queso, quinua, frutas, huevo, pan, carne.

b) Galletas dulces, chocolates, tortas, refrescos de caja.

c) Frugos, leche chocolatada, chisitos

e) Todas las anteriores 
13.- Con respecto al consumo de azúcares, marque lo correcto

a.- El niño nunca debe consumir azúcar

b.- El niño puede consumir azúcar en varios momentos durante el día

c.- El niño puede consumir azúcar en horas determinadas y luego cepillarse los dientes

d.- Todas las anteriores

14.- ¿Hasta qué edad se recomienda dar al niño lactancia materna?

a.- Hasta los 15 días de nacido

b.- Hasta los 3 meses de edad

c.- Hasta los 6 meses de edad

d.- Hasta el primer mes de nacido

15.- Dejar que el niño tenga el biberón con líquidos azucarados durante toda la noche ¿qué causaría?

a.- Va a estar más fuerte al despertar

b.- Estará más fuerte y sano

c.- Estará más expuesto a tener caries

d.- No pasa nada

\section{HIGIENE BUCAL}

16.- ¿Cuántas veces al día el niño debe cepillarse los dientes?

a.- 1 vez

b.- De 2 a 3 veces

c.- De 5 a más veces

d.- Los niños no deben cepillarse los dientes
17.- ¿Desde qué edad se puede usar pasta dental con flúor?

a) A partir de los 2 años

b) A partir de 5 años

c) A partir de la adolescencia

d) En niños menores de 2 años

18.- ¿El cepillado se recomienda realizarlo después de cada comida?

a) No

b) Solo antes de dormir

c) Solo con usar un enjuagatorio basta

d) $\mathrm{Si}$

19.- ¿Cada qué tiempo se debe cambiar el cepillo dental?

a) Cada 3 meses

b) Cada 8 meses

c) $\mathrm{Al}$ año

d) Nunca

20.- En cuanto al cepillado dental

a.- El cepillado dental puede ser realizado solo por el mismo niño

b.- Los padres deben supervisar el cepillado dental de sus niños a esta edad

c.- El cepillado dental debe ser realizado en niños mayores de 3 años.

d.- En el niño el cepillado dental debe realizarse sin pasta dental. 